\title{
A Computational and Experimental Investigation of the Origin of Selectivity in the Chiral Phosphoric Acid Catalyzed Enantioselective Minisci Reaction
}

\author{
Kristaps Ermanis, ${ }^{*}$ Avene C. Colgan, Rupert S. J. Proctor, Barbara W. Hadrys, Robert J. Phipps, ${ }^{*}$ \\ and Jonathan M. Goodman*
}

Cite This: J. Am. Chem. Soc. 2020, 142, 21091-21101

Read Online

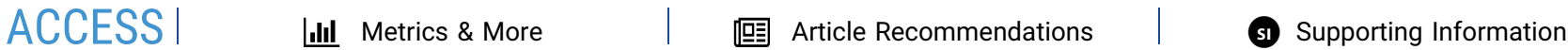

ABSTRACT: The Minisci reaction is one of the most valuable methods for directly functionalizing basic heteroarenes to form carbon-carbon bonds. Use of prochiral, heteroatom-substituted radicals results in stereocenters being formed adjacent to the heteroaromatic system, generating motifs which are valuable in medicinal chemistry and chiral ligand design. Recently a highly enantioselective and regioselective protocol for the Minisci reaction was developed, using chiral phosphoric acid catalysis. However, the precise mechanism by which this process operated and the origin of selectivity remained unclear, making it challenging to develop the reaction more generally. Herein we report further experimental

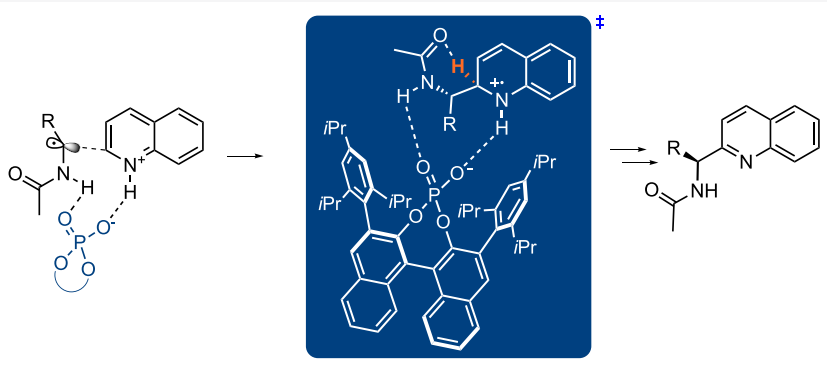
mechanistic studies which feed into detailed DFT calculations that probe the precise nature of the stereochemistry-determining step. Computational and experimental evidence together support Curtin-Hammett control in this reaction, with initial radical addition being quick and reversible, and enantioselectivity being achieved in the subsequent slower, irreversible deprotonation. A detailed survey via DFT calculations assessed a number of different possibilities for selectivity-determining deprotonation of the radical cation intermediate. Computations point to a clear preference for an initially unexpected mode of internal deprotonation enacted by the amide group, which is a crucial structural feature of the radical precursor, with the assistance of the associated chiral phosphate. This unconventional stereodetermining step underpins the high enantioselectivities and regioselectivities observed. The mechanistic model was further validated by applying it to a test set of substrates possessing varied structural features.

\section{INTRODUCTION}

Minisci-type reactions are one of the most direct and versatile methods for forming new $\mathrm{C}-\mathrm{C}$ bonds directly onto basic heteroarenes and are widely used in particular by medicinal chemists. ${ }^{1}$ Initiated by the addition of nucleophilic radicals to the heteroarene $\pi$-system, a new stereocenter is formed adjacent to the heterocyclic framework if a prochiral radical is used. In 2018 one of our groups reported an enantioselective Minisci-type reaction whereby prochiral $N$-Ac- $\alpha$-amino radicals were oxidatively coupled with quinolines and pyridines with high levels of enantiocontrol at the newly formed stereocenter (Figure 1a). ${ }^{2}$ This was enabled by the use of a Chiral Phosphoric Acid (CPA) catalyst in place of the typical achiral Brønsted acid additives used in Minisci reactions. ${ }^{3-5}$ The scope of this transformation has recently been expanded to diazines, together with the development of a predictive model derived through multivariate statistical analysis. ${ }^{6}$ Additionally, isoquinolines have been shown to be viable substrates ${ }^{7}$ and a three-component enantioselective Minisci reaction has been developed using the same catalytic system. ${ }^{8}$ In all but the latter, the initial $N$-Ac- $\alpha$-amino radical is generated from the single electron reduction of amino acid-derived Redox Active Esters (RAEs) using photoredox catalysis, a protocol that was first applied to the Minisci reaction in a non-enantioselective manner by Cheng, Shang, and Fu. ${ }^{9}$ In addition to the high levels of enantioselectivity achieved, it is of note that the CPAcatalyzed Minisci reactions are also highly regioselective, even when performed on substrates that would commonly give mixtures of regioisomers. While studies have been conducted that empirically develop guidelines to aid regioselectivity and survey the effects of fine-tuning the reaction conditions, poor regioselectivity remains one of the longstanding drawbacks of the Minisci reaction. ${ }^{10}$ Several aspects appear to be crucial to enable the unique enantio- and regioselectivity of this reaction.

Received: September 9, 2020

Published: November 30, 2020 


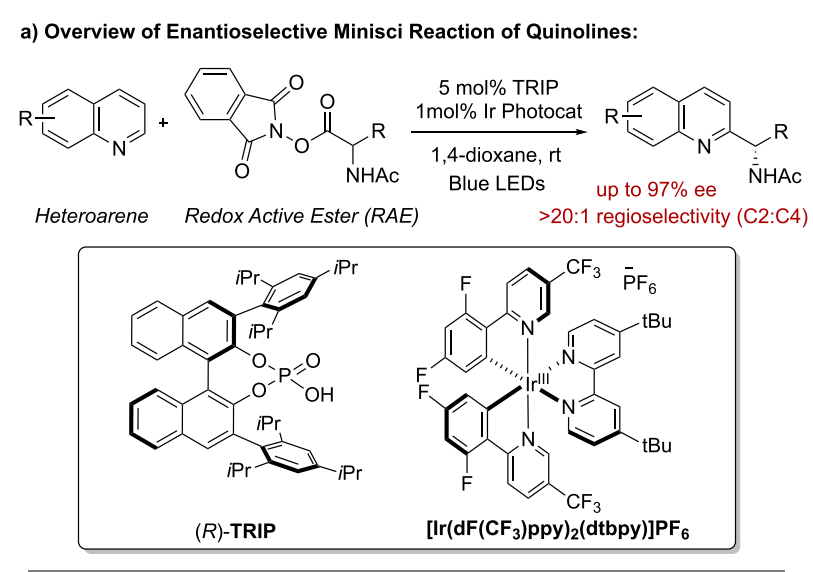

b) Postulated Key Steps in Control of Enantioselectivity:

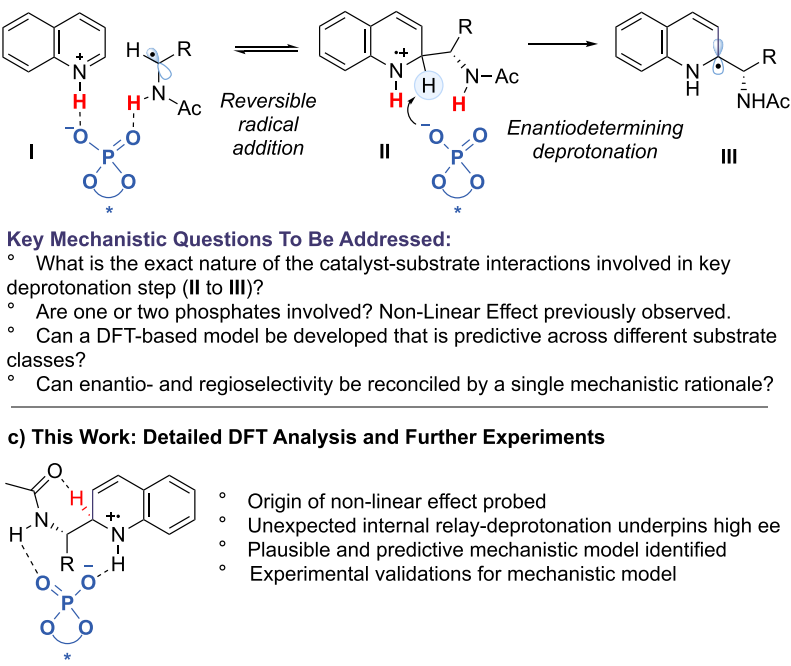

Figure 1. Overview of the enantioselective Minisci reaction, general mechanistic hypothesis, and key questions at the outset of this work.

Firstly, a BINOL-derived CPA catalyst is used to activate the heteroarene to radical addition by protonation, and secondly, the incoming radical nucleophile possesses hydrogen bond donor functionality in the form of an amide, presumably facilitating interaction with the chiral phosphate. Our initial hypothesis was that a network of attractive noncovalent interactions between the protonated heteroarene, bifunctional chiral phosphate, and incoming radical may be conducive to enantiocontrol in one of the ensuing steps of the mechanism. Based on extensive precedent, this mechanism likely consists of initial radical addition to the protonated substrate (Figure 1b, I to II), deprotonation of the resulting radical cation (II to III), and finally single electron oxidation of III to rearomatize the heteroarene (not shown). In principle, enantioinduction could plausibly be envisaged at either the radical addition step or the deprotonation step.

Previous important studies from Minisci and co-workers identified that, for stabilized radicals, the radical addition step is often reversible, as implied by large primary kinetic isotope effects (KIE) for the subsequent deprotonation step. ${ }^{10 a}$ In our original work we observed a primary KIE of 3.6 in an intermolecular competition experiment between quinoline and quinoline- $\mathrm{d}_{7}{ }^{2}$ While the outcome $\left(P_{\mathrm{H}} / P_{\mathrm{D}}\right)$ of this experiment did not provide information on the overall rate-determining step, it strongly suggests a Curtin-Hammett situation, where the enantiodetermining step of the process is deprotonation of the radical cation by the chiral phosphate (Figure 1b, II to III), which would presumably be associated with the quinolinium radical cation through some combination of hydrogen bonding and electrostatic interactions. ${ }^{11}$ The presence of the hydrogen bond donor on the radical fragment was found to be crucial for high ee, suggesting that this component may be interacting with the phosphate during the enantiodetermining deprotonation. While initial mechanistic probes provided some insight, the precise nature of the interactions at the crucial deprotonation step remained elusive. Association of the anionic phosphate with the $\mathrm{NH}$ of the radical cation seemed likely to be maintained throughout. But is it plausible that a single phosphate could interact simultaneously with two hydrogen bond donors (II, highlighted in red) in addition to enacting the crucial deprotonation? To add to this conundrum, we observed a significant, positive nonlinear effect. Although this was just a single, preliminary study, it raised the possibility of two phosphates being involved in the crucial deprotonation event, which could more plausibly account for the multitude of interactions that appeared to be taking place in order to reconcile various observations.

In an attempt to provide answers to these questions we embarked on a detailed study which consists of a full DFT evaluation of the origin of selectivity in the reaction pathway, prefaced by further mechanistic experiments to build on the preliminary ones in our original report. The conclusions from this combined computational and experimental study provide a plausible and predictive mechanistic model for the reaction. The DFT calculations reveal an initially unexpected mode of deprotonation, a relay-deprotonation involving the amide, which accounts excellently for the experimental evidence and addresses many of the questions raised above, which challenged us at the outset (Figure 1c). More broadly, we anticipate that these insights might provide a framework for future applications of chiral phosphate catalysis to radical reactions. Most relevantly, there are now numerous examples of processes where a radical cation, often accessed via photocatalytic single-electron oxidation, ${ }^{12}$ undergoes deprotonation to form a reactive $\alpha$-amino radical. Deprotonation using a chiral base, such as a phosphate, is an emerging strategy for enabling subsequent asymmetric radical chemistry and has been recently showcased by Knowles, Miller and co-workers in the context of a challenging deracemization reaction. ${ }^{4 k}$ We anticipate that this detailed study of the origin of selectivity in the CPA-catalyzed Minisci reaction will raise the awareness of the opportunities afforded by this approach.

\section{RESULTS AND DISCUSSION}

Mechanistic Experiments. In our initial report we carried out two preliminary mechanistic experiments. ${ }^{2}$ The first was an intermolecular kinetic isotope effect (KIE) experiment to interrogate whether deprotonation of the radical cation II is playing a role in stereodetermination. We also carried out a nonlinear effect (NLE) study under the standard reaction conditions to probe the possibility of two molecules of catalyst being involved in the enantiodetermining step. These preliminary experiments were first expanded to provide a firmer foundation for the DFT studies.

The KIE experiment originally reported consisted of an intermolecular competition experiment between quinoline and $\mathrm{d}^{7}$-quinoline under the standard reaction conditions but using the phenylalanine-derived RAE as the limiting reagent. This revealed a primary KIE of 3.6, suggesting that radical addition 
is likely reversible and that deprotonation of radical cation II is selectivity-determining (Scheme 1, left-hand entry). This value

\section{Scheme 1. Competition Experiments To Determine Primary} KIE Values
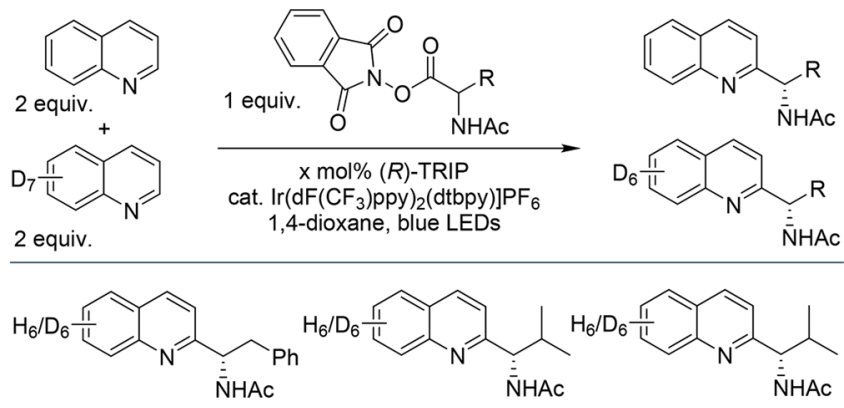

$P h$

dioxane, blue LEDs

$\mathrm{NHAC}$

$5 \mathrm{~mol} \%(R)$-TRIP

$\left[\mathrm{H}_{6}\right] /\left[\mathrm{D}_{6}\right]=3.6$

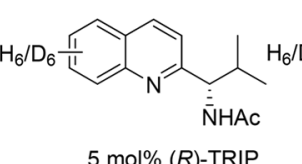

$\left[\mathrm{H}_{6}\right] /\left[\mathrm{D}_{6}\right]=2.9$

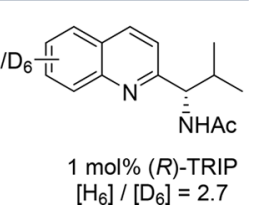

was in agreement with prior studies from Minisci and coworkers who had carried out related experiments examining the addition of stabilized $\alpha$-THF radicals to pyridine and observed a primary KIE of 2.5 in THF for addition at the C2 position. ${ }^{10 a}$ Given that the $\alpha$-amino radicals are also stabilized, our observation of a similar outcome was unsurprising. In the recent three-component CPA-catalyzed Minisci reaction reported by Zheng and Studer, a similar KIE of 2.9 was observed. ${ }^{8}$ To provide further evidence that this was a general feature of the reaction, we carried out an analogous KIE experiment but using the RAE derived from valine, and this gave a similar primary KIE of 2.9 (Scheme 1, middle entry). We also performed the latter experiment but with a lower catalyst loading of $1 \mathrm{~mol} \%$, which gave a similar primary KIE value of 2.7 (Scheme 1, right-hand entry).

Our attention next turned to the NLE experiments and we sought to expand on the single study that had been included in our original report. In that case, a pronounced positive NLE was obtained when the phenylalanine-derived RAE was used with quinoline. ${ }^{2}$ In considering the origin of enantioselectivity, this was potentially a valuable piece of information as the positive NLE could imply the presence of more than one molecule of catalyst in the enantiodetermining step, although it is well-established that there can be other causes for such an observation (vide infra). To obtain a fuller picture we first repeated the NLE experiment with a different radical precursor, the RAE derived from valine (2). Under the same conditions as in the previous study, using $5 \mathrm{~mol} \%$ TRIP catalyst, a positive NLE was again observed (Figure 2, crosses), consistent with that obtained for the RAE derived from phenylalanine. We next repeated the NLE experiment with the valine-derived RAE but using a reduced loading of TRIP (1 mol \% instead of $5 \mathrm{~mol} \%)$. In contrast, the relationship now displayed excellent linearity (Figure 2, diamonds). In light of this we then repeated the phenylalanine-derived RAE at $1 \mathrm{~mol}$ $\%$ and saw a similar outcome-the nonlinear effect disappeared when this lower loading of CPA was used (see Supporting Information (SI)).

Nonlinear effects in asymmetric catalysis can be caused by solubility effects of heterochiral aggregates, giving rise to the so-called "reservoir effect". ${ }^{13}$ The reservoir effect gives rise to positive NLEs and has been shown in the past to be responsible for observed NLEs in several chiral phosphoric acid catalyzed reactions whereby free phosphoric acid forms
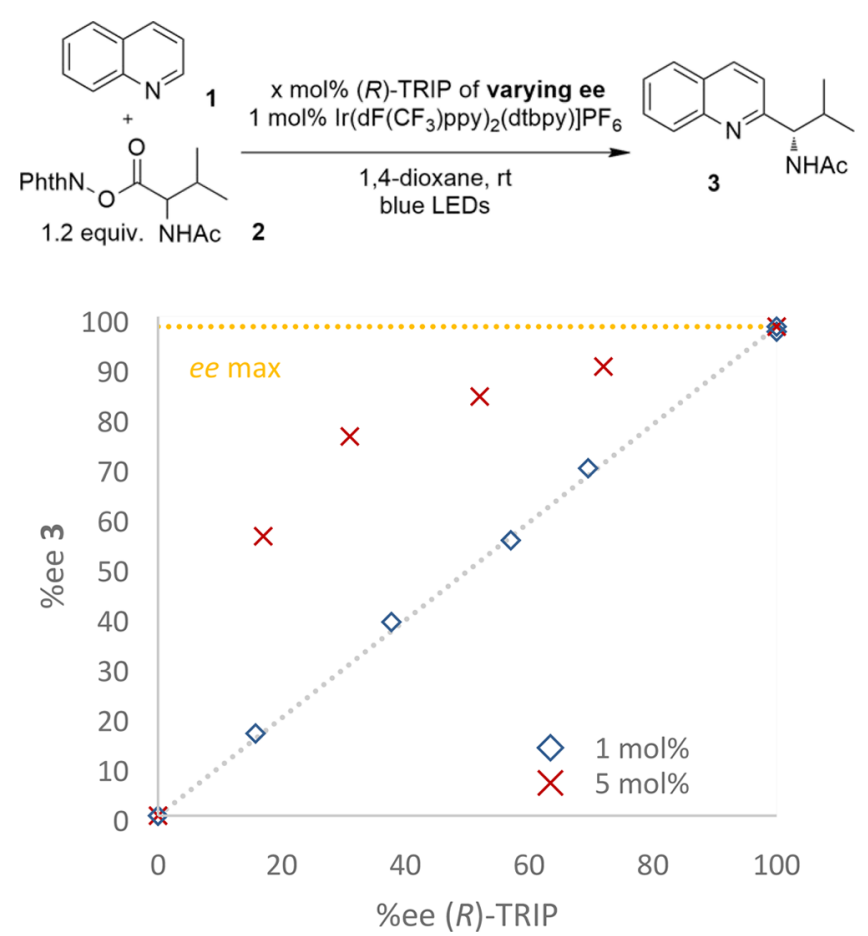

Figure 2. Nonlinear effect studies with the RAE derived from valine, carried out with either $1 \mathrm{~mol} \%$ or $5 \mathrm{~mol} \%$ loading of TRIP. Data points are average of two runs. See SI for full details.

heterochiral dimers which precipitate from nonpolar solvents, such as toluene, provided that there was no basic functionality present to break up such dimers. ${ }^{14}$ It should be noted that in our case the limited solubility of the RAEs meant that reaction mixtures were often heterogeneous, precluding direct observation of any TRIP-dimer precipitates. To further investigate, we sought to determine the extent of protonation of quinoline by $(R)$-TRIP at typical reaction concentration to evaluate to what extent the free phosphoric acid remains in solution and is thus liable to form dimers. An NMR titration experiment in dioxane- $\mathrm{d}_{8}$ was performed in which the ${ }^{31} \mathrm{P}$ NMR chemical shift of the phosphorus atom of TRIP was monitored on addition of aliquots of quinoline. The data were fitted assuming a 1:1 binding ratio, and the obtained $K$ of $27 \mathrm{M}^{-1}$ indicates that, at the concentration the reaction is run, one would expect a 2.7:1 ratio of 1 -TRIP/TRIP at the outset (see SI Figure S5). In light of this indication that free phosphoric is present to a significant degree, we stirred racemic TRIP in dioxane at concentrations consistent with either a $1 \mathrm{~mol} \%$ or 5 mol \% catalyst loading in our reactions. No precipitate was observed in either case after $14 \mathrm{~h}$, providing evidence against the heterochiral dimer precipitation, which accounted for the NLE observed in the previous studies. ${ }^{14}$ We then repeated these experiments with the addition of 1 equiv of quinoline to each. In the $1 \mathrm{~mol} \%$ case, precipitate did form but started to slowly appear only after $2 \mathrm{~h}$ of stirring, a time frame in which the CPA-catalyzed Minisci reaction on this substrate would have proceeded to a significant degree. In contrast, with $5 \mathrm{~mol}$ $\%$ rac-TRIP a precipitate was formed almost immediately. When these latter two experiments were repeated with enantiopure $(R)$-TRIP, no precipitate was formed in either case, even after $14 \mathrm{~h}$ of stirring. These observations strongly suggest that the quinoline-TRIP salt forms a heterochiral aggregate with limited solubility in dioxane and that this is 
a)

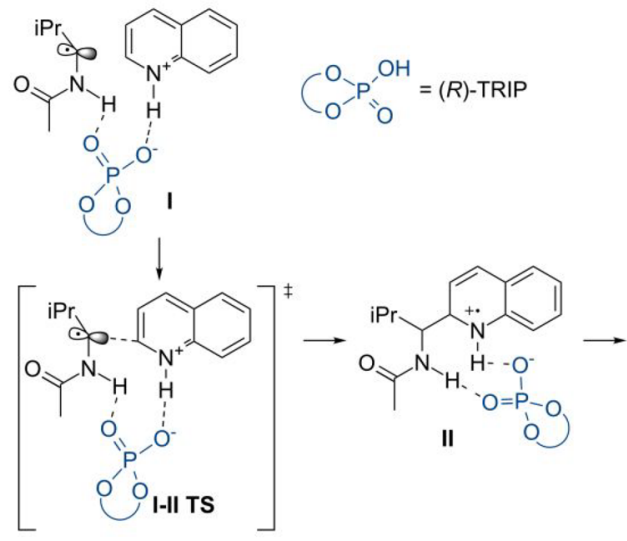
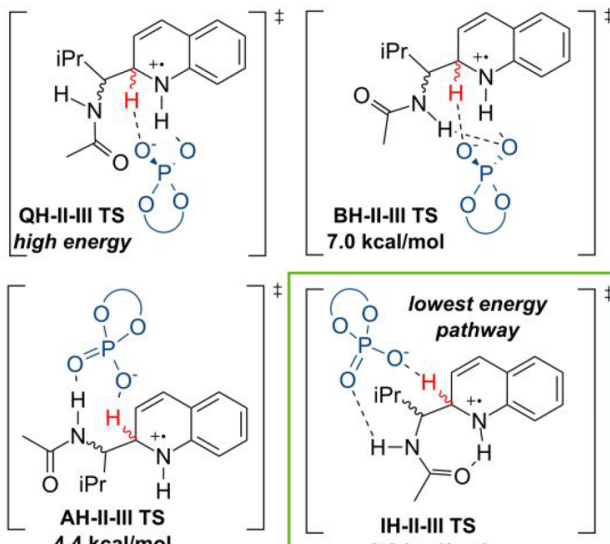
$7.0 \mathrm{kcal} / \mathrm{mo}$

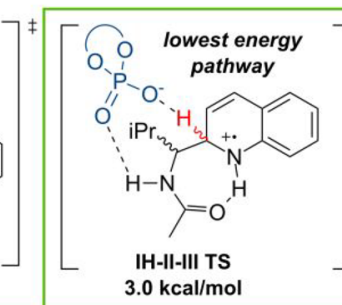

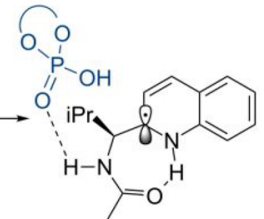

(R)-TRIP.S-III

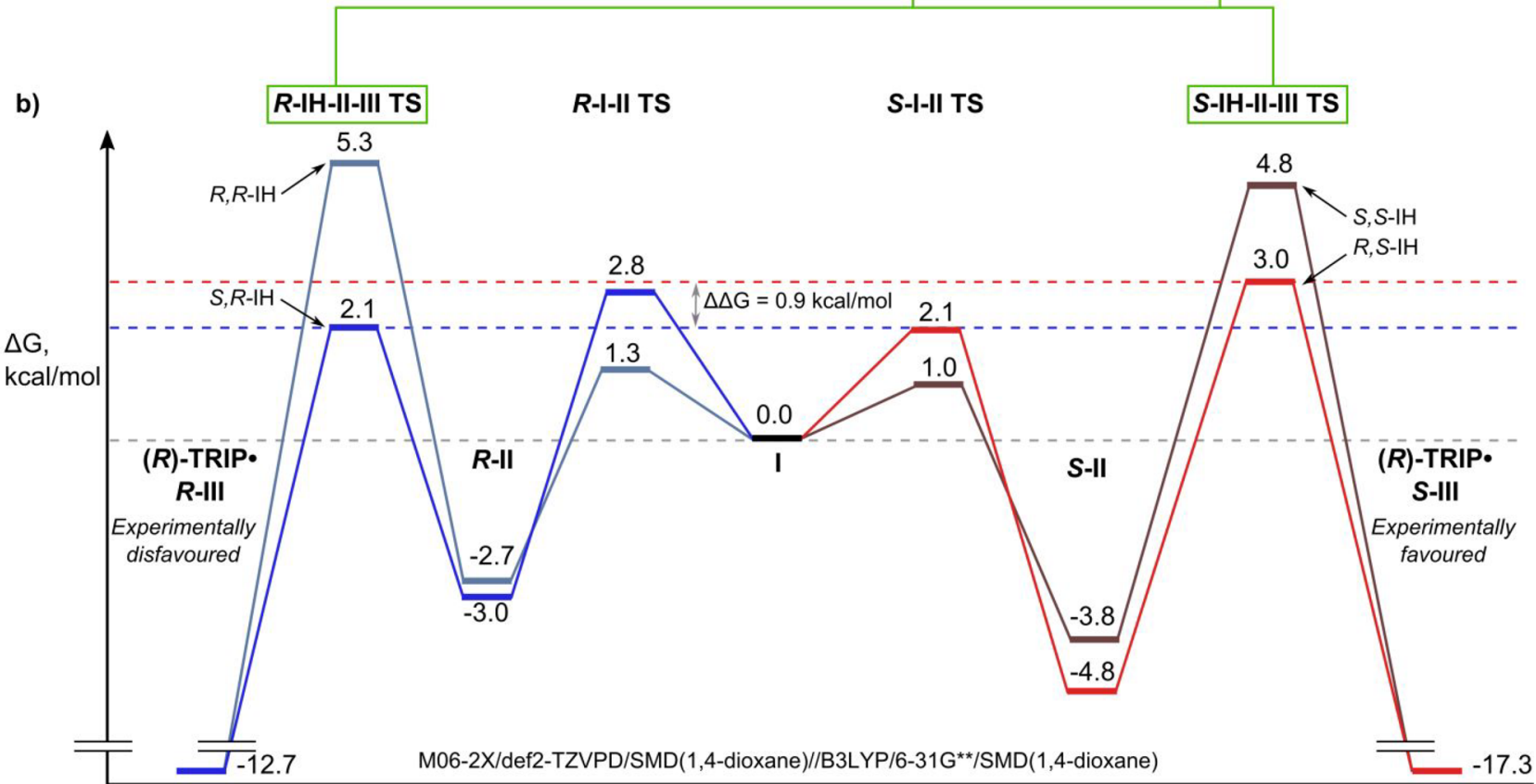

Figure 3. (a) Initial mechanistic hypothesis and four computationally identified deprotonation activation modes: $\mathbf{Q H}, \mathbf{B H}, \mathbf{A H}$, and $\mathbf{I H}$. (b) Full reaction pathway energy diagram via the IH activation mode displaying all four diastereomeric addition transition states (I-II TS) and all four diastereomeric deprotonation transition states (II-III TS). All energies relative to the QuinTRIP/radical complex I; M06-2X/def2-TZVPD/ $\operatorname{SMD}(1,4$-dioxane)//B3LYP/6-31G**/SMD(1,4-dioxane).

responsible for the observed positive NLE when $5 \mathrm{~mol} \%$ of TRIP was used (see SI). Despite efforts, we have so far been unable to obtain X-ray quality crystals of the salt in order to identify its precise structure. Given the above findings it seems unlikely that two molecules of catalyst are involved in the enantiodetermining step of the process, although it cannot be completely ruled out. ${ }^{13 \mathrm{c}}$ We have attempted to obtain kinetic data but have been unable to obtain the necessary reproducibility, even under very carefully controlled conditions and so were not able to explore this avenue further in an experimental manner.

Computational Investigations. The mechanistic experiments outlined above provided a solid foundation for commencing computational studies, which we anticipated would give a more fine-grained mechanistic understanding. ${ }^{15}$ To understand the precise enantioinduction mechanism, the stereochemically relevant steps in the reaction pathway were explored in detail (Figure 3a). The focus of this study is on the origin of selectivity - the reductive fragmentation of RAEs to generate radicals through photoredox catalysis and by other means is well established, and this aspect will not be explored in any detail herein. ${ }^{16}$ The starting point was chosen to be the Quin.TRIP/amino acid derived radical complex I. All of the structures of interest were first submitted to a thorough conformational search. A selection of the resulting geometries were optimized at the $\mathrm{B} \mathrm{LYP}^{17} / 6-31 \mathrm{G}^{* * 18} / \mathrm{SMD}(1,4$-dioxane $)^{19}$ level with M06-2X $\mathrm{X}^{20} /$ def2-TZVPD ${ }^{21} / \operatorname{SMD}(1,4$-dioxane) single-point energy corrections. While these computational methods have been shown to give reliable results in a large variety of systems, we were mindful of the possibility for systematic error in this particular case. It has been shown that DFT methods can overestimate the stability of delocalized radicals compared to localized radicals. ${ }^{22}$ This could impact our results, as in the proposed reaction mechanism a localized radical in $\mathbf{I}$ is transformed into a highly delocalized radical cation in II. Hence we were aware that direct comparison of 

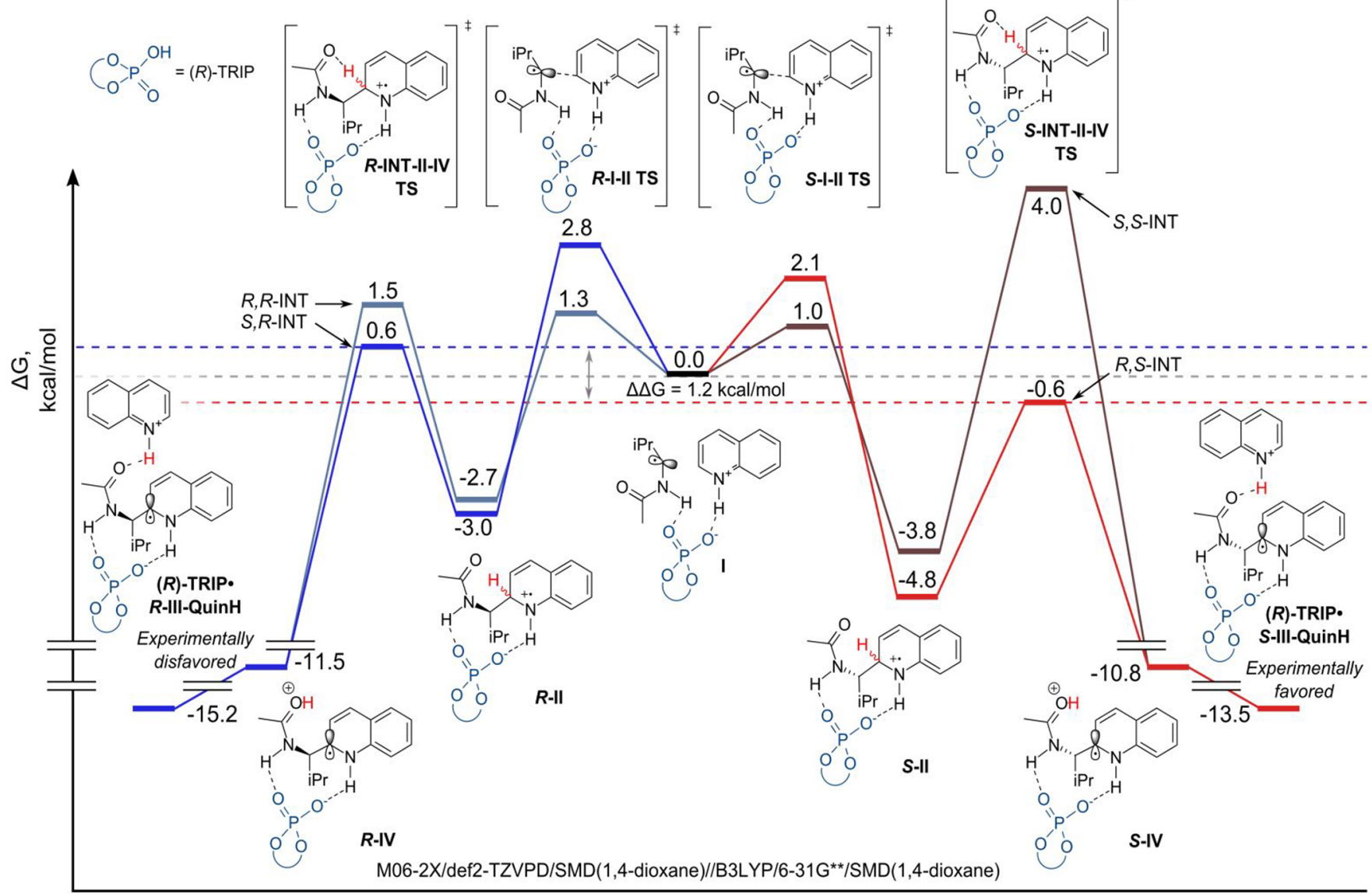

Figure 4. Revised full reaction pathway energy diagram via the computationally identified novel deprotonation mode INT displaying all four diastereomeric addition transition states (I-II TS) and all four diastereomeric deprotonation transition states (II-IV TS). All energies relative to the QuinTRIP/radical complex I; M06-2X/def2-TZVPD/SMD(1,4-dioxane)//B3LYP/6-31G**/SMD(1,4-dioxane).

the energies of these intermediates or transition states involving them should be approached with caution. The KIE experiments strongly suggested that deprotonation of the radical cation was the product-determining step, and so we proceeded on the assumption of a Curtin-Hammett scenario, where the relative barriers of this deprotonation would determine the enantioselectivity outcome.

The radical addition (I-II) was found to have low barriers of between 1.0 and $2.8 \mathrm{kcal} / \mathrm{mol}$ for the four diastereomeric addition transition states (not shown), and the resulting radical cation intermediates II were all $4-7 \mathrm{kcal} / \mathrm{mol}$ downhill from the addition transition state of each.

A thorough exploration of the possible deprotonation (IIIII) modes was then undertaken, with the minimal assumption that the TRIP-phosphate acts as the base. Four different modes were identified: $\mathbf{Q H}, \mathbf{B H}, \mathbf{A H}$, and $\mathbf{I H}$ (Figure 3a). $\mathbf{Q H}$ features a strong hydrogen bond between the phosphate and the $\mathrm{NH}$ of the quinolinum radical cation, but no interaction with the amide. It was significantly higher than all other modes in our preliminary studies and was not examined in further detail. BH forms two hydrogen bonds between a single phosphate oxygen and both the substrate amide and quinolinium $\mathrm{NH}$ and was found to have activation energies around $7 \mathrm{kcal} / \mathrm{mol}$, relative to the starting complex. AH features a strong hydrogen bond between the amide $\mathrm{NH}$ and has a free energy of only $4.4 \mathrm{kcal} / \mathrm{mol}$ above the starting complex I. This mode of deprotonation has been used to explain the enantioselectivity in this reaction in a recently reported concurrent DFT study of the same reaction. ${ }^{23}$ However, on further exploration we identified IH, which features an internal hydrogen bond between the amide carbonyl and the quinolinium $\mathrm{NH}$, as the lowest energy activation mode at $3.0 \mathrm{kcal} / \mathrm{mol}$, leading to the experimentally favored $S$ product $(\boldsymbol{R}, S$-IH, Figure $3 \mathrm{~b})$. The resulting reaction profile was also consistent within DFT error margin with a reversible addition and product-determining deprotonation. However, the competing transition state $S, R-I H$ leading to the $R$ product was found to be $0.9 \mathrm{kcal} / \mathrm{mol}$ lower than the $S$ transition state and, therefore, predicted the enantioselectivity opposite to that which was experimentally observed, assuming deprotonation is the product-determining step.

The failure of these four deprotonation modes prompted us to reassess and consider more unconventional modes. After laborious exploration (see SI for full details) we ultimately discovered a lower energy deprotonation mode, INT, in which the amide, itself activated by the phosphate, acts as an internal base to perform the deprotonation (Figure 4). This effectively constitutes a "switched" form of IH in which the amide and the phosphate exchange roles. The resulting protonated amide IV is then in turn deprotonated by an external quinoline base in a barrierless and highly favorable process. The lowest $R, S$-INT internal deprotonation transition state has free energy $3.6 \mathrm{kcal} /$ mol lower than the IH mode. Furthermore, it is $5.0 \mathrm{kcal} / \mathrm{mol}$ lower than the AH-type activation mode that was invoked in 
the previously reported DFT study of this reaction. ${ }^{23}$ Upon full exploration, this unexpected INT mode also gave an accurate prediction of enantioselectivity. The enantiomeric $S, R$-INT mode leading to the experimentally minor $R$ product had a relative free activation energy $1.2 \mathrm{kcal} / \mathrm{mol}$ higher, consistent with the experimentally observed enantioselectivity of $94 \%$ ee, if deprotonation is the product-determining step.

In fact, our computations predicted INT deprotonation to be so low in energy relative to the starting complex and the addition transition states that this raised doubts about whether it could be the stereodetermining step (Figure 4). As this is contrary to the results from the KIE experiments, this aspect was investigated in more detail. First, computational prediction of KIE outcomes for both addition and deprotonation steps was performed, replacing quinoline with quinoline- $\mathrm{d}_{7}$ to match the experiments. While hybridization change at a deuterated center can give measurable $\mathrm{KIE},{ }^{24}$ calculations clearly showed that no KIE should be expected in the addition step. In contrast, a KIE value of $\sim 5$ was predicted for the deprotonation via the INT mode, which is consistent with the experimentally observed values $(2.7-3.6)$.

Both experimental and computational KIE values pointed to the deprotonation as being the stereodetermining step. Similarly, the INT mechanism is so much lower in energy than the alternatives that it seemed highly likely to be the actual deprotonation mechanism. That left a systematic error in DFT barrier heights for addition and deprotonation as a possible explanation. In the reaction mechanism, a localized radical in the starting complex $\mathbf{I}$ is converted into a highly delocalized aromatic radical in the addition (II) and deprotonation product (III, via IV). As mentioned previously, DFT methods are known to overestimate the stability of delocalized radicals compared to localized radicals. ${ }^{22}$ This means that the energies of all of the deprotonation steps should be trustworthy when compared with each other, but the deprotonation process may require a systematic correction to be applied in order to compare the calculated energies to those for the addition process. To test this, we explored the reaction mechanism using a higher level double-hybrid B2PLYPD3 method. ${ }^{25}$ B2PLYPD3 has been shown to be a generalpurpose, higher-accuracy, and more expensive computational method and should exhibit much reduced artifacts of this nature. As this is a significantly more expensive method with worse scaling characteristics, a model system with a simplified catalyst (biphenyl-2,2'-diyl phosphate; see SI section 3) was used. The model system qualitatively reproduced the key features of the full-system behavior, including the relative energetic ordering of the addition I-II transition states, intermediates II, and deprotonation II-III and II-IV transition states. However, the higher level B2PLYPD3 single-point energies for the model system show a clear systematic difference in energies at every step as the radical delocalization increases (see SI Figure S3.2c). These calculations confirmed low radical addition barriers, and even lower barriers for the reverse reaction, strongly suggesting equilibrating radical adducts. Notably, all of the deprotonation transition states, including $R, S$-INT, are now higher in energy than the addition transition states. This confirmed our suspicion that the INT deprotonation mechanism is the stereodetermining step, despite the initial DFT results suggesting otherwise. Importantly, there is still close agreement in relative energies within each step between the M06-2X and B2PLYPD3 results for the two diastereomeric pathways and the various activation modes. This enables us to use the much more practical B3LYP and M06-2X functionals to predict and understand the enantioselectivity in CPA-catalyzed Minisci reactions. It must also be noted that while INT is the stereodetermining step, previous computational work indicates that the overall rate-determining step of the reaction is the radical generation with the highest barrier of $19.0 \mathrm{kcal} / \mathrm{mol}-$ much higher than the INT deprotonation barriers identified here. $^{23}$

Understanding the INT Deprotonation Pathway. We next examined the DFT results in more detail to understand what makes the INT pathway so efficient and selective. While the deprotonation can proceed via four different diastereomeric pathways, there is a clear preference for the $R, S$ and $S, R$ pathways, which lead to the major and minor product enantiomer, respectively. An explanation for this preference can be deduced from the lowest energy INT transition state geometries, which are half chair-like (Figure 5a). Both $R, S$ and

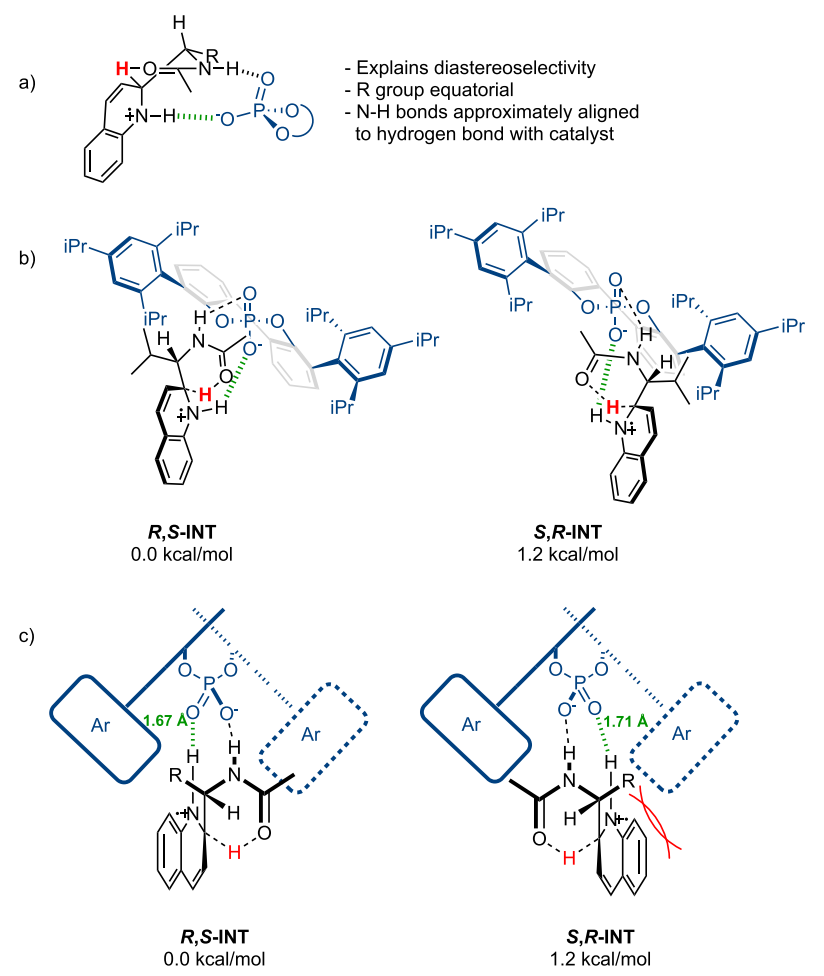

Figure 5. Rationalizing the stereoselectivity in the INT deprotonation mode. (a) Diastereoselectivity model, (b) enantioselectivity models (front view), and (c) enantioselectivity models (top view).

$S, R$ allow the substituent $\mathrm{R}$ to take the more favorable equatorial position, while in the $S, S$ and $R, R$ pathways $\mathrm{R}$ is forced to occupy the unfavorable axial position. The enantioselectivity seems to be provided by the subtle combination of steric and hydrogen bonding interactions (Figure $5 \mathrm{~b}$ ). In the lowest energy, minor $R$ enantiomerproducing transition state $S, R-I N T$, the hydrogen bond between quinolinium $\mathrm{NH}$ and phosphate oxygen, is longer (1.71 $\AA$ vs $1.67 \AA$ in $R, S$-INT) and also the quinolinium $\mathrm{N}-\mathrm{H}$ bond is bent significantly more out of the quinolinium plane $\left(8.1^{\circ}\right.$ vs $3.1^{\circ}$ in $R, S$-INT). Finally, in the $S, R$-INT transition state a steric clash between the quinolinium ring and the large $3,3^{\prime}$-substituent on the CPA catalyst pushes the quinolinium $\mathrm{NH}$ away from the phosphate, distorting the hydrogen bond 
and contributing to the energy difference between the two transition states (Figure 5c).

While these considerations explain the catalyst selectivity for only one of the four possible diastereomeric pathways, they do not necessarily explain its activity. As the INT pathway is an internal deprotonation, in principle it could occur outside of the catalyst pocket. However, the uncatalyzed INT process has a barrier $3.8 \mathrm{kcal} / \mathrm{mol}$ higher than the catalyzed version. In an enzyme-like fashion, a major constituent of this catalytic effect appears to be reactive conformation stabilization. For the uncatalyzed pathway, the isolated radical cation intermediate prefers to form an internal hydrogen bond between the amide carbonyl and the quinolinium proton-this bent conformation is favored by $4.0 \mathrm{kcal} / \mathrm{mol}$ compared to the INT-like extended conformation (Figure 6a). In contrast, this preference is

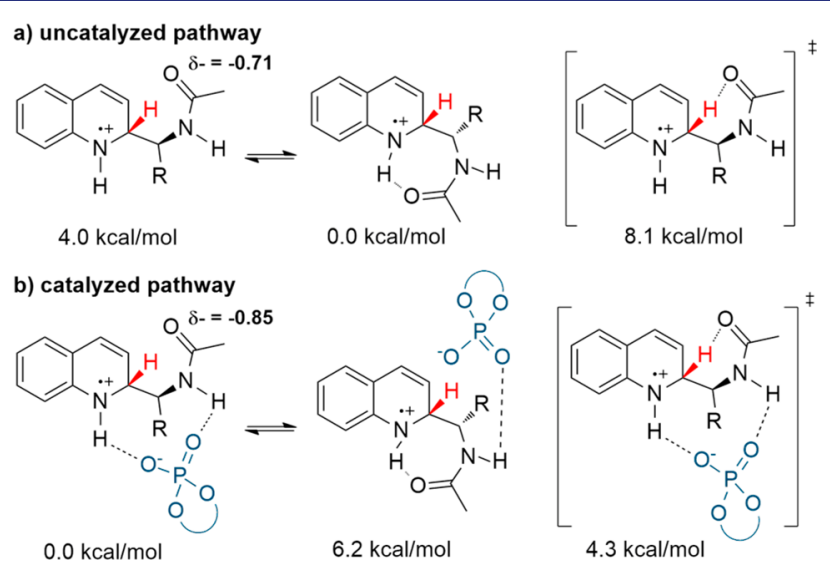

Figure 6. Comparing the conformational equilibria outside (a) and inside (b) the catalyst pocket. All energies are free energies relative to the lowest energy intermediate conformation; M06-2X/def2TZVPD/SMD(1,4-dioxane)//B3LYP/6-31G**/SMD(1,4-dioxane).

completely reversed inside the catalyst pocket as the INT-like conformation is stabilized by two strong hydrogen bonds with the phosphate catalyst (Figure 6b). Another important effect is that the hydrogen bonds between the substrate and the phosphate catalyst also make the amide carbonyl more basic through the inductive effect. This is clearly demonstrated by Mulliken charges - the free intermediate carbonyl oxygen has a charge of -0.71 , while the catalyst bound intermediate has a significantly more negative charge of -0.85 on the oxygen. ${ }^{26}$

Regioselectivity. As mentioned previously, the CPAcatalyzed Minisci reaction is notable for its outstanding regioselectivity outcomes, particularly on quinolines, which are notorious for giving mixtures of $\mathrm{C} 2$ - and $\mathrm{C} 4$ - products in most protocols. The outcome of this study that the INT pathway is highly favored also sheds revealing light on this selectivity aspect. The very low energy of the internal deprotonation pathway is contingent on two strong hydrogen bonds being formed between the chiral phosphate and the substrate. If radical addition occurs at the $\mathrm{C} 4$ position, such an arrangement would simply be impossible to achieve due to the distance to the quinolinium $\mathrm{NH}$, meaning that only one hydrogen bond would be feasible during the deprotonation transition state. The energy of this could be expected to be more akin to that of $\mathbf{A H}$, the only mode considered herein in which there is no hydrogen bond to the $\mathrm{NH}$ of the quinolinium radical cation and whose energy was significantly higher than both the INT and IH modes, which each contain two. During the extensive scope studies on quinolines, we had never observed even trace amounts of the $\mathrm{C} 4$ product under the optimized conditions, an observation that correlates very satisfactorily with the present mechanistic hypothesis.

Benchmarking of the Computational Model. Having established a plausible origin of enantioselectivity for the CPAcatalyzed Minisci reaction using a single substrate, we sought to further test its generality and predictive ability when applied to a range of substrates in which each component was varied. The next lowest deprotonation mechanism IH was chosen for comparison to the preferred INT pathway that emerged from the detailed study. The two mechanisms exhibit very different substrate orientations in the catalyst pocket, and therefore the substrate tolerance would be presumed to be quite different depending on which mode is at play. In addition, IH gave a lower and opposite enantioselectivity prediction in our initial studies.

Based on these substrate scope considerations, we selected four additional substrates (5-8, Figure 7). These encompassed several steric variations of the RAE as well as variations of the heteroarene to include an extended $\pi$ system (6) and a second heteroatom $(7,8)$. For each substrate, deprotonation via both the IH and INT mode was thoroughly explored using DFT methods.

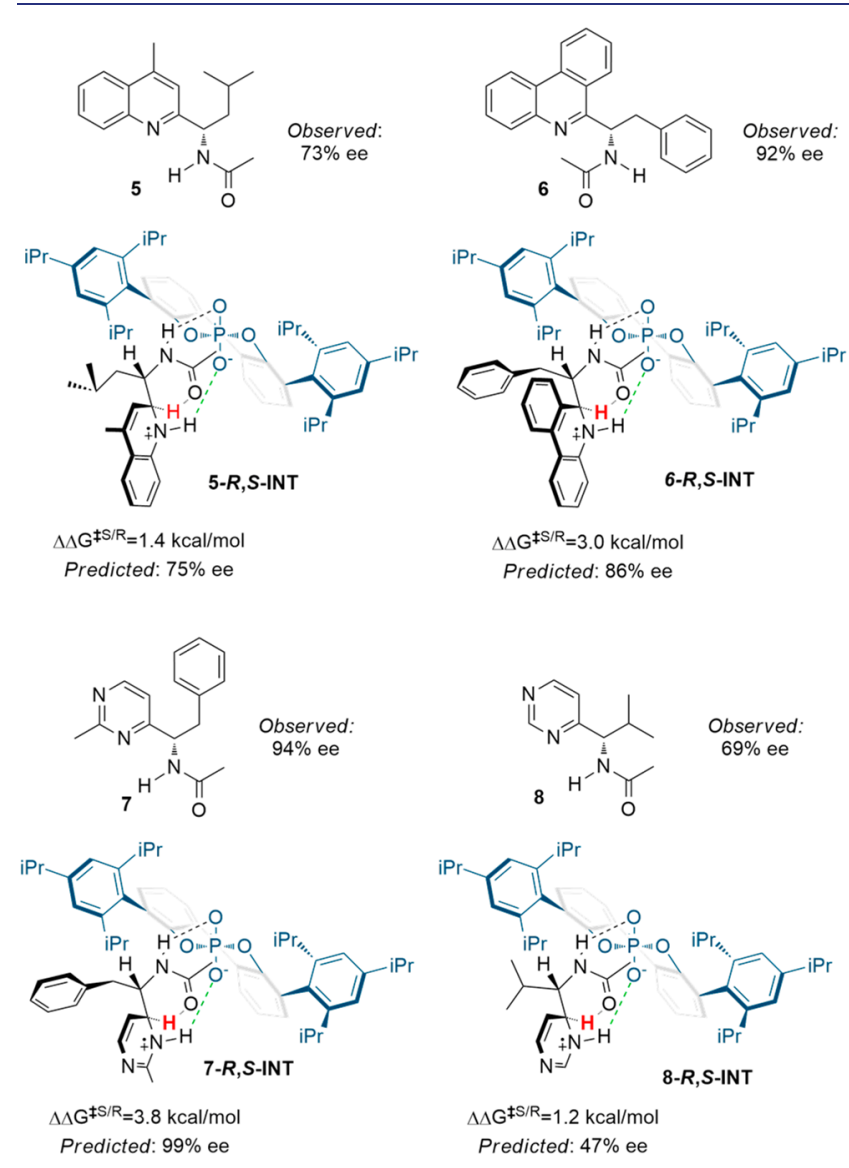

Figure 7. Additional substrates explored computationally with experimentally observed enantioselectivities, and the computationally located lowest energy deprotonation transition states. The energies quoted are the difference between the lowest energy $S$ and $R$ producing INT transition states, M06-2X/def2-TZVP/SMD(1,4dioxane)// B3LYP/6-31G**. The enantioselectivity predictions take into account both INT and IH deprotonation modes. 
The results from the INT mode alone are depicted alongside each substrate in Figure 7 and typically show very good predictive capability. We specifically included in the selection two substrates which had given somewhat lower ee values experimentally ( $\mathbf{5}$ and $\mathbf{8}$ ), and indeed the modeling predicted these effectively, in addition to the high ee examples (6 and 7). The calculated ee values for the INT mode are shown alongside those for the IH mode in Table 1 and emphasize

Table 1. Comparison of the Computational Enantioselectivity Prediction Results from Different Mechanisms on Various Substrates ${ }^{a}$

\begin{tabular}{cccccc} 
& & \multicolumn{3}{c}{ Predicted (\% ee) } & \\
\cline { 3 - 5 } Substrate & $\begin{array}{c}\text { Number of TSs } \\
\text { explored }\end{array}$ & IH & INT & IH+INT & $\begin{array}{c}\text { Experimental } \\
(\% \text { ee })\end{array}$ \\
\cline { 3 - 5 } 3 & 26 & 56 & 89 & 89 & 94 \\
$\mathbf{5}$ & 37 & -50 & 83 & 75 & 73 \\
$\mathbf{6}$ & 40 & -65 & 96 & 86 & $\mathbf{9 2}$ \\
7 & 43 & 10 & 99 & 99 & $\mathbf{9 4}$ \\
$\mathbf{8}$ & 31 & 78 & 46 & 46 & $\mathbf{6 9}$ \\
MAE & & $\mathbf{8 2}$ & $\mathbf{9}$ & $\mathbf{8}$ &
\end{tabular}

${ }^{a}$ Energies and enantioselectivity predictions based on results from M06-2X/def2-TZVP/SMD(1,4-dioxane)//B3LYP/6-31G**. MAE: mean absolute enantioselectivity prediction error for each mechanism.

how poor the IH mode is in a predictive sense. The mean absolute error (MAE) for enantioselectivity predictions across substrates was $82 \%$ ee for IH, while INT was an order of magnitude more accurate with an MAE of only $9 \%$ ee. Furthermore, the energies with $\mathbf{I H}$ were always predicted to be significantly higher than the INT mode. The predictions made using INT could be slightly refined further by adjusting for the fact that IH and INT deprotonation pathways may be occurring concurrently.

The R,S-INT deprotonation mode was found to be the most energetically favorable in all five substrates, providing further support to the generality of this mechanistic model (Figure 7).

These computational studies, along with the extensive previous experimental results, ${ }^{2-5}$ allow us to suggest general guidelines about the substrate scope of enantioselective CPAcatalyzed Minisci reactions (Figure 8). Quinolinium NH, the amide $\mathrm{NH}$, and the amide carbonyl functionality are all critical

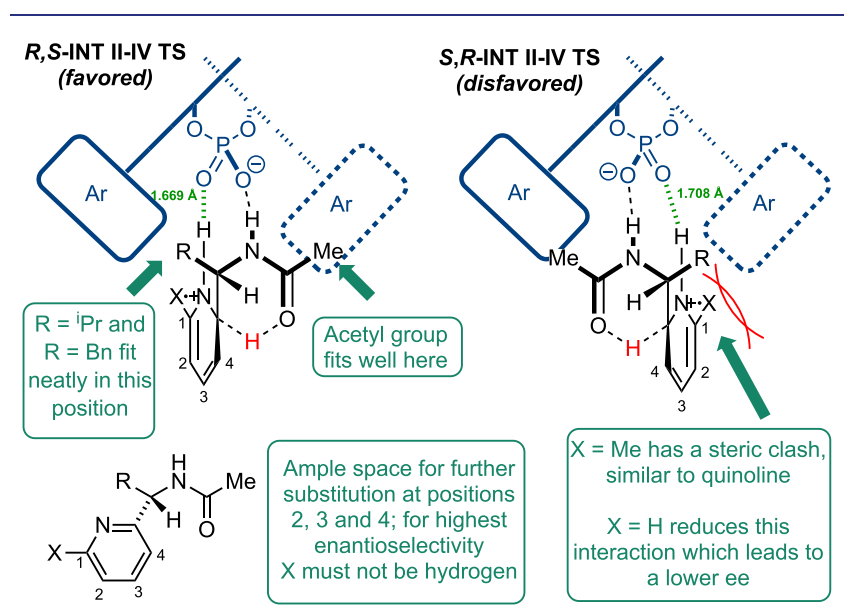

Figure 8. Rationalization of prior experimental observations relating to scope, in the context of the new computational model. for the efficient and selective INT activation mode, and substrates lacking any of these showed worse reactivity and/or selectivity. This accounts for the observation in our previous study that a proline-derived RAE, lacking the amide $\mathrm{NH}$, gave poor selectivity. Similarly, $N$-Boc and $N$-Cbz $\alpha$-amino radicals gave very poor selectivity in our original optimization studies, and this also is readily explained by the INT mechanism, as the amide carbonyl should be significantly less basic in these substrates. ${ }^{2}$

As the aromatic ring of the heteroarene is projecting out of the CPA catalyst pocket, a broad range of substitution patterns are typically tolerated on the heteroarene, particularly at positions 2, 3, and 4 (Figure 8). A substituent larger than $\mathrm{H}$ is beneficial at position $1(\mathrm{X})$, as this leads to unfavorable steric interactions with the lower (as depicted) aryl ring of TRIP in the disfavored $S, R$-INT transition state, increasing enantioselectivity. This interaction explains why quinolines are typically excellent substrates for this reaction. Finally, for the substituent $\mathrm{R}$ on the RAE both small and moderate steric bulk are tolerated, as there is sufficient space between them and the CPA 3,3'-substituent in the INT transition state. Larger substituents, such as iPr and Bn, can also be tolerated if they are flexible and are able to rotate out of the way of the CPA $3,3^{\prime}$-substituent. Indeed, the recently developed statistical model describing enantioselectivity in this reaction contained a negative term for the length of the substituent $\mathrm{R}$ on the RAE, meaning that increasing the length of $\mathrm{R}$ leads to reduced enantioselectivity. The origin of this trend now becomes clear. ${ }^{6}$

Overall Reaction Mechanism. In summary, the experimental and computational studies reported here, taking into account previous reported evidence, allow us to build a much more detailed picture of selectivity control in the CPAcatalyzed Minisci reaction (Figure 9). The radical generated from reduction of the RAE interacts with the Quin.TRIP complex in $\mathbf{I}$ and adds to the quinolinium via one of four possible diastereomeric transition states (I-II TS). The addition is reversible, allowing deprotonation of radical cation II to eventually proceed through the lowest energy $R, S$-INT mechanism. In this unconventional deprotonation mechanism, the carbonyl oxygen of the $\mathrm{N}$-Acetyl group serves as an internal base, assisted by the phosphate (II-IV TS). The protonated carbonyl of IV is in turn deprotonated by an external quinoline molecule, neutralizing the substrate and allowing its dissociation from the phosphate catalyst. Finally, the dissociated, neutral radical III undergoes single-electron oxidation, resulting in product formation after proton loss. While most studies of Minisci reactions involving photoredox catalysis propose that this oxidation step is part of the photoredox cycle, ${ }^{9,27}$ chain processes are also possible ${ }^{28}$ and direct HAT has been proposed. ${ }^{23}$

\section{CONCLUSIONS}

Our computational and experimental investigation of the origin of selectivity in the CPA-catalyzed Minisci reaction have established that the Curtin-Hammett principle is in operation: a fast and reversible radical addition is followed by a slower, irreversible enantioselective deprotonation which determines enantioselectivity in the product. The investigations led to the discovery of a selectivity-determining pathway through an initially unexpected internal mode of deprotonation, $R, S$-INT II-III TS (Figure 9). DFT calculation of the energy of this structure provides a quantitative insight into the selectivity of the process, which is consistent with all of our experimental 


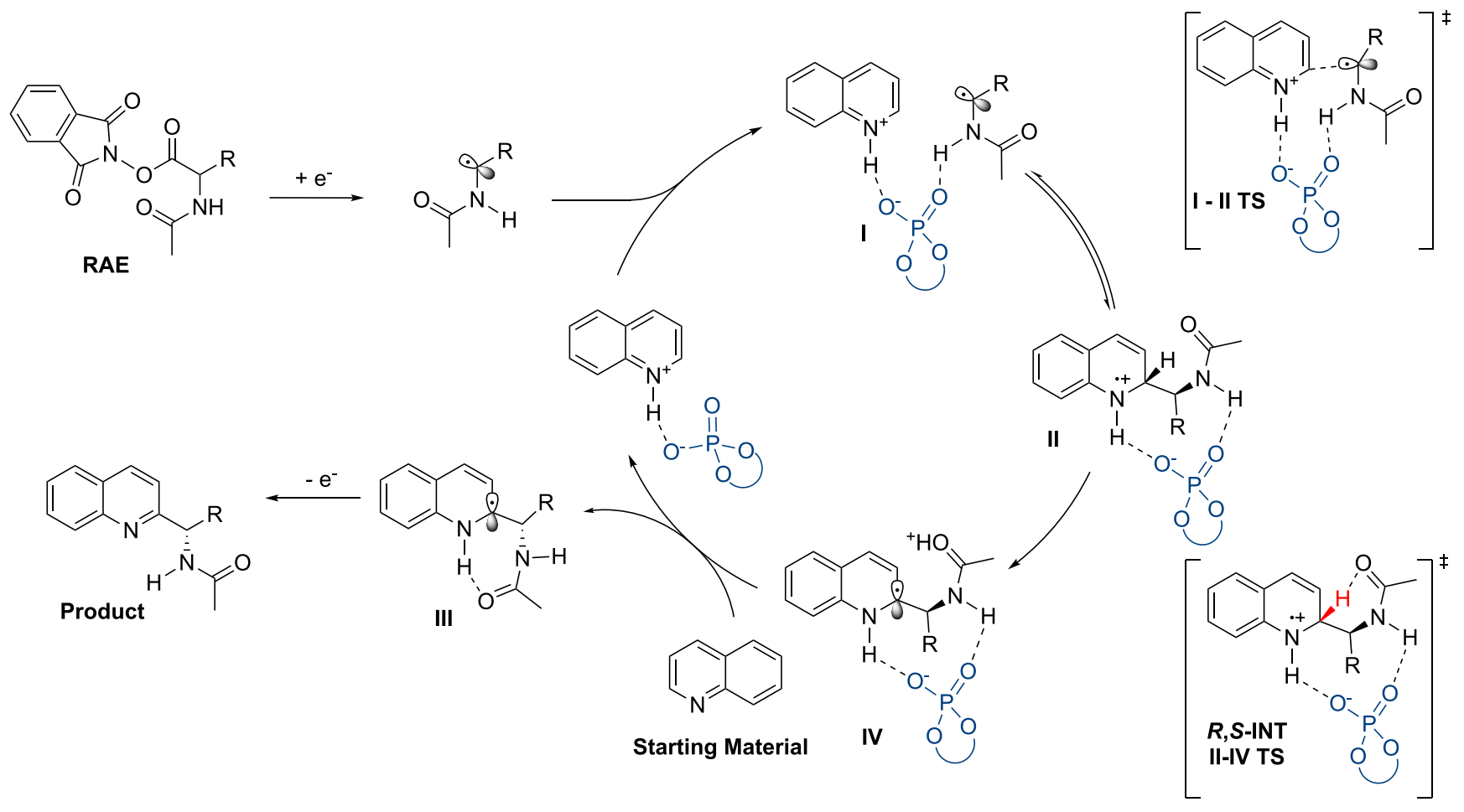

Figure 9. Overview of selectivity control in the CPA-catalyzed Minisci reaction.

and computational data. The structure also provides a qualitative explanation for both the degree and the sense of the high levels of regioselectivity and enantioselectivity that are observed in the reaction. We anticipate that these findings may allow a wider variety of enantioselective Minisci reaction types to be developed. More broadly, we hope that they may influence the design of future enantioselective radical reactions using versatile chiral phosphoric acid catalysts.

\section{ASSOCIATED CONTENT}

\section{(s) Supporting Information}

The Supporting Information is available free of charge at https://pubs.acs.org/doi/10.1021/jacs.0c09668.

Experimental details, procedures, compound characterization data, and computational details (PDF)

\section{AUTHOR INFORMATION}

\section{Corresponding Authors}

Kristaps Ermanis - Department of Chemistry, University of Cambridge, Cambridge CB2 1EW, United Kingdom;

Email: ke291@cam.ac.uk

Robert J. Phipps - Department of Chemistry, University of Cambridge, Cambridge CB2 1EW, United Kingdom; ○ orcid.org/0000-0002-7383-5469; Email: rjp71@ cam.ac.uk

Jonathan M. Goodman - Department of Chemistry, University of Cambridge, Cambridge CB2 1EW, United Kingdom; orcid.org/0000-0002-8693-9136;

Email: jmg11@cam.ac.uk

\section{Authors}

Avene C. Colgan - Department of Chemistry, University of Cambridge, Cambridge CB2 1EW, United Kingdom
Rupert S. J. Proctor - Department of Chemistry, University of Cambridge, Cambridge CB2 1EW, United Kingdom; (1) orcid.org/0000-0002-2296-448X

Barbara W. Hadrys - Department of Chemistry, University of Cambridge, Cambridge CB2 1EW, United Kingdom

Complete contact information is available at:

https://pubs.acs.org/10.1021/jacs.0c09668

\section{Notes}

Full set of DFT output files with optimized structures, frequencies and high-level single-point energies can be found at 10.17863/CAM.56880.

The authors declare no competing financial interest.

\section{ACKNOWLEDGMENTS}

We are grateful to the EPSRC and GlaxoSmithKline for $\mathrm{PhD}$ studentships (to R.S.J.P. and B.W.H.), the Royal Society for a University Research Fellowship (to R.J.P.), the Leverhulme Trust (RPG-2018-081), and the European Research Council (Starting Grant 757381, NonCovRegioSiteCat). We also thank the Leverhulme Trust (ECF-2017-255) and the Isaac Newton Trust (17.08(d)) for an Early Career Fellowship (to K.E.). The computational work has been performed using resources provided by the Cambridge Tier-2 system operated by the University of Cambridge Research Computing Service (http:// www.hpc.cam.ac.uk) funded by EPSRC Tier-2 Capital Grant EP/P020259/1. We are grateful to Tim Barrett (GlaxoSmithKline) for useful discussions.

\section{REFERENCES}

(1) (a) Minisci, F.; Vismara, E.; Fontana, F. Recent Developments of Free-Radical Substitutions of Heteroaromatic Bases. Heterocycles 1989, 28, 489-519. (b) Minisci, F.; Fontana, F.; Vismara, E. Substitutions by nucleophilic free radicals: A new general reaction of heteroaromatic bases. J. Heterocycl. Chem. 1990, 27, 79-96. 
(c) Duncton, M. A. J. Minisci reactions: Versatile $\mathrm{CH}$-functionalizations for medicinal chemists. MedChem Comm 2011, 2, 1135-1161. (d) Tauber, J.; Imbri, D.; Opatz, T. Radical Addition to Iminium Ions and Cationic Heterocycles. Molecules 2014, 19, 16190. (e) Proctor, R. S. J.; Phipps, R. J. Recent Advances in Minisci-Type Reactions. Angew. Chem., Int. Ed. 2019, 58, 13666-13699.

(2) Proctor, R. S. J.; Davis, H. J.; Phipps, R. J. Catalytic enantioselective Minisci-type addition to heteroarenes. Science 2018, 360, 419-422.

(3) (a) Uraguchi, D.; Terada, M. Chiral Brønsted Acid-Catalyzed Direct Mannich Reactions via Electrophilic Activation. J. Am. Chem. Soc. 2004, 126, 5356-5357. (b) Akiyama, T.; Itoh, J.; Yokota, K.; Fuchibe, K. Enantioselective Mannich-Type Reaction Catalyzed by a Chiral Brønsted Acid. Angew. Chem., Int. Ed. 2004, 43, 1566-1568. (c) Parmar, D.; Sugiono, E.; Raja, S.; Rueping, M. Complete Field Guide to Asymmetric BINOL-Phosphate Derived Brønsted Acid and Metal Catalysis: History and Classification by Mode of Activation; Brønsted Acidity, Hydrogen Bonding, Ion Pairing, and Metal Phosphates. Chem. Rev. 2014, 114, 9047-9153.

(4) For examples of CPAs in radical chemistry, see: (a) Rono, L. J.; Yayla, H. G.; Wang, D. Y.; Armstrong, M. F.; Knowles, R. R. Enantioselective Photoredox Catalysis Enabled by Proton-Coupled Electron Transfer: Development of an Asymmetric Aza-Pinacol Cyclization. J. Am. Chem. Soc. 2013, 135, 17735-17738. (b) Lin, J.S.; Dong, X.-Y.; Li, T.-T.; Jiang, N.-C.; Tan, B.; Liu, X.-Y. A DualCatalytic Strategy To Direct Asymmetric Radical Aminotrifluoromethylation of Alkenes. J. Am. Chem. Soc. 2016, 138, 9357-9360. (c) Hepburn, H. B.; Melchiorre, P. Brønsted acid-catalysed conjugate addition of photochemically generated $\alpha$-amino radicals to alkenylpyridines. Chem. Commun. 2016, 52, 3520-3523. (d) Wang, F.-L.; Dong, X.-Y.; Lin, J.-S.; Zeng, Y.; Jiao, G.-Y.; Gu, Q.-S.; Guo, X.Q.; Ma, C.-L.; Liu, X.-Y. Catalytic Asymmetric Radical Diamination of Alkenes. Chem. 2017, 3, 979-990. (e) Lin, L.; Bai, X.; Ye, X.; Zhao, X.; Tan, C.-H.; Jiang, Z. Organocatalytic Enantioselective Protonation for Photoreduction of Activated Ketones and Ketimines Induced by Visible Light. Angew. Chem., Int. Ed. 2017, 56, 13842-13846. (f) Li, J.; Kong, M.; Qiao, B.; Lee, R.; Zhao, X.; Jiang, Z. Formal enantioconvergent substitution of alkyl halides via catalytic asymmetric photoredox radical coupling. Nat. Commun. 2018, 9, 2445. (g) Gentry, E. C.; Rono, L. J.; Hale, M. E.; Matsuura, R.; Knowles, R. R. Enantioselective Synthesis of Pyrroloindolines via Noncovalent Stabilization of Indole Radical Cations and Applications to the Synthesis of Alkaloid Natural Products. J. Am. Chem. Soc. 2018, 140, 3394-3402. (h) Yin, Y.; Dai, Y.; Jia, H.; Li, J.; Bu, L.; Qiao, B.; Zhao, X.; Jiang, Z. Conjugate Addition-Enantioselective Protonation of NAryl Glycines to $\alpha$-Branched 2-Vinylazaarenes via Cooperative Photoredox and Asymmetric Catalysis. J. Am. Chem. Soc. 2018, 140, 6083-6087. (i) Morse, P. D.; Nguyen, T. M.; Cruz, C. L.; Nicewicz, D. A. Enantioselective counter-anions in photoredox catalysis: The asymmetric cation radical Diels-Alder reaction. Tetrahedron 2018, 74, 3266-3272. (j) Cao, K.; Tan, S. M.; Lee, R.; Yang, S.; Jia, H.; Zhao, X.; Qiao, B.; Jiang, Z. Catalytic Enantioselective Addition of Prochiral Radicals to Vinylpyridines. J. Am. Chem. Soc. 2019, 141, 5437-5443. (k) Shin, N. Y.; Ryss, J. M.; Zhang, X.; Miller, S. J.; Knowles, R. R. Light driven deracemization enabled by excited state electron transfer. Science 2019, 366, 364-369. (1) Lin, J.-S.; Li, T.-T.; Liu, J.-R.; Jiao, G.Y.; Gu, Q.-S.; Cheng, J.-T.; Guo, Y.-L.; Hong, X.; Liu, X.-Y. Cu/Chiral Phosphoric Acid-Catalyzed Asymmetric Three-Component RadicalInitiated 1,2-Dicarbofunctionalization of Alkenes. J. Am. Chem. Soc. 2019, 141, 1074-1083. (m) Roos, C. B.; Demaerel, J.; Graff, D. E.; Knowles, R. R. Enantioselective Hydroamination of Alkenes with Sulfonamides Enabled by Proton-Coupled Electron Transfer. J. Am. Chem. Soc. 2020, 142, 5974-5979. (n) Ye, L.; Tian, Y.; Meng, X.; Gu, Q.-S.; Liu, X.-Y. Enantioselective Copper(I)/Chiral Phosphoric Acid Catalyzed Intramolecular Amination of Allylic and Benzylic C-H Bonds. Angew. Chem., Int. Ed. 2020, 59, 1129-1133.

(5) Proctor, R. S. J.; Colgan, A. C.; Phipps, R. J. Exploiting attractive non-covalent interactions for the enantioselective catalysis of reactions involving radical intermediates. Nat. Chem. 2020, 12, 990-1004.
(6) Reid, J. P.; Proctor, R. S. J.; Sigman, M. S.; Phipps, R. J. Predictive Multivariate Linear Regression Analysis Guides Successful Catalytic Enantioselective Minisci Reactions of Diazines. J. Am. Chem. Soc. 2019, 141, 19178-19185.

(7) Liu, X.; Liu, Y.; Chai, G.; Qiao, B.; Zhao, X.; Jiang, Z. Organocatalytic Enantioselective Addition of $\alpha$-Aminoalkyl Radicals to Isoquinolines. Org. Lett. 2018, 20, 6298-6301.

(8) Zheng, D.; Studer, A. Asymmetric Synthesis of Heterocyclic $\gamma$ Amino-Acid and Diamine Derivatives by Three-Component Radical Cascade Reactions. Angew. Chem., Int. Ed. 2019, 58, 15803-15807.

(9) Cheng, W.-M.; Shang, R.; Fu, Y. Photoredox/Brønsted Acid CoCatalysis Enabling Decarboxylative Coupling of Amino Acid and Peptide Redox-Active Esters with N-Heteroarenes. ACS Catal. 2017, 7, 907-911.

(10) (a) Minisci, F.; Vismara, E.; Fontana, F.; Morini, G.; Serravalle, M.; Giordano, C. Polar effects in free-radical reactions. Solvent and isotope effects and effects of base catalysis on the regio- and chemoselectivity of the substitution of protonated heteroaromatic bases by nucleophilic carbon-centered radicals. J. Org. Chem. 1987, 52, 730-736. (b) O'Hara, F.; Blackmond, D. G.; Baran, P. S. RadicalBased Regioselective $\mathrm{C}-\mathrm{H}$ Functionalization of Electron-Deficient Heteroarenes: Scope, Tunability, and Predictability. J. Am. Chem. Soc. 2013, 135, 12122-12134. (c) Hadrys, B. W.; Phipps, R. J. Acid and Solvent Effects on the Regioselectivity of Minisci-Type Addition to Quinolines Using Amino Acid Derived Redox Active Esters. Synlett 2020. DOI: $10.1055 / \mathrm{s}-0040-1707888$.

(11) Simmons, E. M.; Hartwig, J. F. On the Interpretation of Deuterium Kinetic Isotope Effects in C-H Bond Functionalizations by Transition-Metal Complexes. Angew. Chem., Int. Ed. 2012, 51, 30663072.

(12) (a) Narayanam, J. M. R.; Stephenson, C. R. J. Visible light photoredox catalysis: applications in organic synthesis. Chem. Soc. Rev. 2011, 40, 102-113. (b) Schultz, D. M.; Yoon, T. P. Solar synthesis: prospects in visible light photocatalysis. Science 2014, 343, 1239176. (c) Shaw, M. H.; Twilton, J.; MacMillan, D. W. C. Photoredox Catalysis in Organic Chemistry. J. Org. Chem. 2016, 81, 6898-6926. (d) Staveness, D.; Bosque, I.; Stephenson, C. R. J. Free Radical Chemistry Enabled by Visible Light-Induced Electron Transfer. Acc. Chem. Res. 2016, 49, 2295-2306.

(13) (a) Girard, C.; Kagan, H. B. Nonlinear Effects in Asymmetric Synthesis and Stereoselective Reactions: Ten Years of Investigation. Angew. Chem., Int. Ed. 1998, 37, 2922-2959. (b) Kagan, H. B. Practical Consequences of Non-Linear Effects in Asymmetric Synthesis. Adv. Synth. Catal. 2001, 343, 227-233. (c) Satyanarayana, T.; Abraham, S.; Kagan, H. B. Nonlinear Effects in Asymmetric Catalysis. Angew. Chem., Int. Ed. 2009, 48, 456-494.

(14) (a) Li, N.; Chen, X.-H.; Zhou, S.-M.; Luo, S.-W.; Song, J.; Ren, L.; Gong, L.-Z. Asymmetric Amplification in Phosphoric Acid Catalyzed Reactions. Angew. Chem., Int. Ed. 2010, 49, 6378-6381. (b) Guo, W.; Luo, Y.; Sung, H. H.; Williams, I. D.; Li, P.; Sun, J. Chiral Phosphoric Acid Catalyzed Enantioselective Synthesis of alphaTertiary Amino Ketones from Sulfonium Ylides. J. Am. Chem. Soc. 2020, 142, 14384-14390.

(15) Reid, J. P.; Simón, L.; Goodman, J. M. A Practical Guide for Predicting the Stereochemistry of Bifunctional Phosphoric Acid Catalyzed Reactions of Imines. Acc. Chem. Res. 2016, 49, 1029-1041.

(16) Niu, P.; Li, J.; Zhang, Y.; Huo, C. One-Electron Reduction of Redox-Active Esters to Generate Carbon-Centered Radicals. Eur. J. Org. Chem. 2020, 36, 5801-5814.

(17) (a) Becke, A. D. Density-functional exchange-energy approximation with correct asymptotic behavior. Phys. Rev. A: At., Mol., Opt. Phys. 1988, 38, 3098-3100. (b) Lee, C.; Yang, W.; Parr, R. G. Development of the Colle-Salvetti correlation-energy formula into a functional of the electron density. Phys. Rev. B: Condens. Matter Mater. Phys. 1988, 37, 785-789. (c) Becke, A. D. Density-functional thermochemistry. III. The role of exact exchange. J. Chem. Phys. 1993, $98,5648-5652$.

(18) (a) Hehre, W. J.; Ditchfield, R.; Pople, J. A. Self-Consistent Molecular Orbital Methods. XII. Further Extensions of Gaussian- 
Type Basis Sets for Use in Molecular Orbital Studies of Organic Molecules. J. Chem. Phys. 1972, 56, 2257-2261. (b) Hariharan, P. C.; Pople, J. A. The influence of polarization functions on molecular orbital hydrogenation energies. Theor. Chim. Acta. 1973, 28, 213222.

(19) Marenich, A. V.; Cramer, C. J.; Truhlar, D. G. Universal Solvation Model Based on Solute Electron Density and on a Continuum Model of the Solvent Defined by the Bulk Dielectric Constant and Atomic Surface Tensions. J. Phys. Chem. B 2009, 113, 6378-6396.

(20) Zhao, Y.; Truhlar, D. G. The M06 suite of density functionals for main group thermochemistry, thermochemical kinetics, noncovalent interactions, excited states, and transition elements: two new functionals and systematic testing of four M06-class functionals and 12 other functionals. Theor. Chem. Acc. 2008, 120, 215-241.

(21) Rappoport, D.; Furche, F. Property-optimized Gaussian basis sets for molecular response calculations. J. Chem. Phys. 2010, 133, 134105 .

(22) Guerra, M. Overestimation of the stability of the $\pi$-delocalized versus the $\sigma$-localized configuration in radicals by current density functionals: the case of vinylacyl radicals. Theor. Chem. Acc. 2000, 104, 455-460.

(23) Feng, A.; Yang, Y.; Liu, Y.; Geng, C.; Zhu, R.; Zhang, D. Mechanism and Origins of Enantio- and Regioselectivities in Catalytic Asymmetric Minisci-Type Addition to Heteroarenes. J. Org. Chem. 2020, 85, 7207-7217.

(24) Singleton, D. A.; Thomas, A. A. High-Precision Simultaneous Determination of Multiple Small Kinetic Isotope Effects at Natural Abundance. J. Am. Chem. Soc. 1995, 117, 9357-9358.

(25) (a) Grimme, S. Semiempirical hybrid density functional with perturbative second-order correlation. J. Chem. Phys. 2006, 124, 034108. (b) Pakhira, S.; Sen, K.; Sahu, C.; Das, A. K. Performance of dispersion-corrected double hybrid density functional theory: A computational study of OCS-hydrocarbon van der Waals complexes. J. Chem. Phys. 2013, 138, 164319.

(26) Mulliken, R. S. Electronic Population Analysis on LCAO-MO Molecular Wave Functions. I. J. Chem. Phys. 1955, 23, 1833-1840.

(27) (a) Jin, J.; MacMillan, D. W. C. Direct $\alpha$-Arylation of Ethers through the Combination of Photoredox-Mediated C-H Functionalization and the Minisci Reaction. Angew. Chem., Int. Ed. 2015, 54, 1565-1569. (b) Huff, C. A.; Cohen, R. D.; Dykstra, K. D.; Streckfuss, E.; DiRocco, D. A.; Krska, S. W. Photoredox-Catalyzed Hydroxymethylation of Heteroaromatic Bases. J. Org. Chem. 2016, 81, 69806987. (c) Li, G.-X.; Morales-Rivera, C. A.; Wang, Y.; Gao, F.; He, G.; Liu, P.; Chen, G. Photoredox-mediated Minisci C-H alkylation of Nheteroarenes using boronic acids and hypervalent iodine. Chem. Sci. 2016, 7, 6407-6412. (d) Garza-Sanchez, R. A.; Tlahuext-Aca, A.; Tavakoli, G.; Glorius, F. Visible Light-Mediated Direct Decarboxylative $\mathrm{C}-\mathrm{H}$ Functionalization of Heteroarenes. ACS Catal. 2017, 7, 4057-4061. (e) Sherwood, T. C.; Li, N.; Yazdani, A. N.; Dhar, T. G. M. Organocatalyzed, Visible-Light Photoredox-Mediated, One-Pot Minisci Reaction Using Carboxylic Acids via N-(Acyloxy)phthalimides. J. Org. Chem. 2018, 83, 3000-3012.

(28) (a) Cheng, W.-M.; Shang, R.; Fu, M.-C.; Fu, Y. PhotoredoxCatalysed Decarboxylative Alkylation of N-Heteroarenes with $\mathrm{N}$ (Acyloxy)phthalimides. Chem. - Eur. J. 2017, 23, 2537-2541.

(b) Kammer, L. M.; Rahman, A.; Opatz, T. Molecules 2018, 23, 764. 